\title{
Editorial: Bringing New Music to New Audiences
}

\section{INTRODUCTION}

This issue of Organised Sound focuses on an event that took place in September 2018 under the auspices of the Creative Europe-funded 'Interfaces' project (www.interfacesnetwork.eu). One of this visionary project's actions was a three-day conference focused on best-practice models for making a wide variety of forms of new music more accessible in terms of appreciation and participation than they are currently. Clearly many of the presentations and presented works focused on instrumental/vocal facilitation that fall outside the field of this journal. What has been selected for this issue consists of a number of presentations, workshops and artworks (performance works and installations) that formed part of the conference that was called 'Bringing New Music to New Audiences', essentially the driving theme of the Interfaces project. Associated with this theme, one article has been included, written by the project director, Christos Carras, that was originally presented at a conference at the Onassis Cultural Centre (Stegi) in Athens where he is executive director and head of the music programme. Issue $24 / 3$ is completed by one off-thematic submission and a book review.

This editorial will first introduce Interfaces briefly followed by an overview of the conference and its affiliated website hub. Following this the issue's articles will be summarised.

\section{INTERFACES}

The Interfaces project, which runs from 2016 to 2020, consists of the following partners:

- The Onassis Cultural Centre (Athens) - lead partner

- De Montfort University: Music, Technology and Innovation - Institute for Sonic Creativity

- European University Cyprus (Nicosia)

- Ictus (Ensemble, Brussels)

- IRCAM (Paris)

- Klangforum Wien (Ensemble, Vienna)

- Q-O2 (Brussels)

- ZKM (Karlsruhe)

On the project website, Interfaces - New Models and Practices of Audience Development in Contemporary
Music in Europe, the project's full title, is described as follows:

Interfaces is an international, interdisciplinary project focusing on bringing new music to an extensive range of new audiences. It involves a partnership of organisations from a wide range of European countries having a broad spectrum of experience in fields such as performing, multi-media exhibitions, new media, acoustic and electroacoustic research and education. This trans-sectoral approach is the key to opening up new perspectives on both the creative dimension of the project and the central objective, which is to engage new audiences of all ages and those potential audience segments which, for a variety of demographic or cultural reasons have not yet been exposed to the music of our time.

Actions range from new performance formats in new innovative spaces and across artistic disciplines using new media for creation and dissemination to educational activities including physical outreach and innovative online applications to research on audience drivers and conferences to artistic residencies. Its objectives consequently include audience development, transnational mobility and capacity building. Its vision and rich information concerning all its actions can be found on the above-mentioned website.

\section{BRINGING NEW MUSIC TO NEW AUDIENCES}

The conference's homepage can be found at interfaces. dmu.ac.uk/conference/, which leads to links to the full conference programme, booklet and more. It took place on 21-3 September 2018 in Leicester, UK. Its original announcement summarises its vision (the tense has been changed):

\footnotetext{
'Bringing New Music to New Audiences' was a three-day international conference which was intended to bring together community artists and other musicians, educators, animateurs, specialists within music and other cultural organisations, government policy representatives and representatives from cross-cultural projects who are involved with initiatives related to the conference title. Of course, project participants, non-academics and others were also most welcome.
}

By 'new music' what is meant is original innovative works of music, including the sonic arts, which largely reside 
outside the commercial sector. The goal was to share and debate different forms of good practice relating to how new music can be used as the means of engaging with new communities and ways through which new music can reach underrepresented communities.

The conference focused on a selection of community arts and pedagogical initiatives related to participation and community action. It offered diverse workshops and performances and introduced conference participants to a wide range of programmes within this area. Subjects included, but were not limited to:

- educational initiatives

- working with community organisations

- the musical equivalent of public art

- interculturalism.

- ways of effectively and robustly measuring/evaluating impact and research projects regarding the above.

[The conference] is related to the 'Bringing New Music to New Audiences' resource site www.interfaces.dmu.ac.uk/ hub which is [intended] to offer information regarding good practice in outreach initiatives around the globe. All interested parties are welcome to send their work to that hub at any time once made available.

The conference keynote was Susanna Eastburn, chief executive of Sound and Music (UK).

The papers selected here were based on quality and relevance to the issue. Although, as was the case in terms of submissions for the event itself, there are quite a few UK-based authors, thematic diversity formed another criterion for selection.

\section{THIS ISSUE 24/3}

The launch article for this issue was a presentation based on one of the more unexpected submissions received. The article focuses on workshops taking place in rural Tumbisca, some $35 \mathrm{~km}$ from Morelia, the capital of Michoacán state in Mexico at a school covering the age group of 6 to 15 year olds. Similar to other submissions below, the goal here is to create a learning environment for children enabling electroacoustic music creativity. Mario Alberto DuarteGarcía along with Jorge Rodrigo Sigal-Sefchovich describe some of the challenges as well as contextualise how this project was developed and share a selection of the project results.

A UK example follows involving work that forms part of Interfaces, one focused on sound-based creativity workshops for children and for people of all ages. (Another related action focused on DIY making in schools - see www.interfaces.dmu.ac.uk/activities/ hacking-and-sound-based-creativity-in-schools/ and www.interfaces.dmu.ac.uk/activities/diy-instruments-inschools/.) Inspired by De Montfort's Compose with
Sounds and EARS 2 initiatives (see, for example, Organised Sound 18/2), a series of workshops has been placed on DMU's Interfaces site which David Holland and Duncan Chapman have offered in the UK and around Europe and others are now using internationally. The article discusses the path that led towards the creation of these workshops and the methods involved and includes statistical data on evaluative feedback received based on the institute's Intention/Reception methodology illustrating a very high engagement rate thus far upon workshop completion.

The third item in this issue also involves new software developments. In this case, Rodolphe Bourotte and Sharon Kanach discuss a major step in the history of Iannis Xenakis's UPIC, namely the creation of UPISketch, something demonstrated at the conference and co-developed as part of Interfaces with the European University Cyprus. Like UPIC, users draw on a surface, in this case tablets, where a great deal of parameters and sound samples are already embedded into the program. The article presents the pedagogical ideas behind UPISketch and discusses early workshops during which this new product was first presented.

The fourth article in this collection takes a step away from the conference whilst introducing Interfaces project director Christos Carras's vision regarding how ' $[\mathrm{t}$ ]he diverse practice of soundwalking is approached through its constituent parts (walking and listening) as an ideal "way in" to the appreciation of new sonic art'. Carras looks into this development both historically and philosophically offering a holistic context to support his vision. Key to this vision is participation, something that is a common denominator of all other thematic submissions to this issue. The author illustrates his concepts citing work of three practitioners all of whom have presented soundwalks in urban Athens.

Another conference focus was investigating the theme from the educational studies point of view. Where all other presentations came from people incorporating outreach into their work in music, in the case of Motje Wolf and Sarah Younie, the goal was to investigate how the theme could be supported from the point of view of pedagogical studies. Their point of departure is that many if not most teachers, whether music specialists or more generalist teachers at primary level, will be unfamiliar with sound-based creativity and thus need a helping hand to enable them to support students' learning. In other words enabling is here focused on teachers as opposed to any particular musical community. Their text investigates the development of teachers' packs for the above-mentioned EARS 2 project as well as the creation of a so-called MESHGuide for sonic creativity where MESH stands for Mapping Education Specialist knowHow, in both 
cases involving the creation of materials to support the conference theme through teaching at primary and secondary school levels in this field.

Barbara Lüneburg offers a very different slant on the conference theme. Her project, presented as a talk and associated concert performance, is entitled "TransCoding - From "Highbrow Art" to Participatory Culture'. As project leader she worked with a number of people online representing a variety of backgrounds, most of whom had roots in today's popular culture. They worked together to create multimedia artworks involving projections, instruments and any sounds. Communication took place by way of the project's social media hub challenging traditional means of artistic production. Although it was clear here that professional and non-expert participants were involved, the goal was to find new means of collaborative creative expression. The author presents information regarding the means of production including discussions regarding some of the hurdles encountered. One subject, common to most initiatives regarding widening participation, was that of empowerment. This can be a challenge: what she has discovered is that to achieve a successful collaboration, at times personal practices have to be discarded in order to achieve a common group goal. Lüneburg thus offers a balanced overview of a project that challenged many artistic norms.

A second practice-based submission is from architectmusician Emma-Kate Matthews who presented work in which spatial music presentation was seen as a means of aiding the development of interest for new music (in her case both electroacoustic and instrumental). Here a focus is on immersion and spatial awareness, in particular in terms of how sounds change location or move around a space. During her presentation, different images illustrated sonic position whilst listeners heard the multichannel sound spatialisation. Access was at least partially achieved through the audiovisual relationships. Although not featured, this geometric connection is one in which both of Matthews's strengths, spatial and sonic design, come to light. She seeks therefore in this article to answer a question posed in her introduction: 'can designers of [performance] spaces do more to facilitate engagement with experimental music?'

Peter Batchelor presented the latest installation work in his GRID series, installations involving many loudspeakers within a contained space. As his title suggests, his goal is the achievement of 'intimate immensity' therefore applying some of Matthews's considerations of engagement by way of an immersive sonic experience. In Batchelor's case this means applying acousmatic techniques within what is normally considered a sound art context. Batchelor discusses how intimacy as an aesthetic experience is relevant to the conference theme whilst also supporting the notion that installations are an excellent access tool in terms of supporting increased accessibility regarding sonic creativity. His ability to have his immersive installations flow from real-world to more abstract sonic material is just one devise used in order to assist listeners in terms of navigating their way through time-based sonic works outside the concert hall.

The issue concludes with an off-theme item and a book review. The final article submission is Maxence Larrieu's contribution, 'A Consideration of the Code of Computer Music as Writing, and Some Thinking on Analytical Theories', in which the author encourages thought about the medium of computer music as differentiated from electroacoustic music due to the use of code over audio signal. The case is made for code's position as an analysable material akin to musical scores, drafts and sketches. The discussion flows around the differences of analyses 'from the signal' (audio signal) and 'before the signal' (code, sketches, interview, etc.), and demonstrates some challenges of terminology such as 'tape music', which Larrieu suggests are insufficient nomenclature 'slowing down' the consideration of computer music code. A concluding remark states that if 'we understand computer music as a written one, it can open new directions for studying it'.

\section{A FINAL WORD}

The term community music - now often replaced by participatory music - has been around for decades and, as a consequence of the excellent work of people working in facilitating access to the arts, many funding organisations strongly support the notion encapsulated in the conference's title. Still, in the area of new music and, in particular, electroacoustic music there have been few such gatherings to share different approaches to supporting access and, to the best of our knowledge, no other online hubs exist gathering examples of good practice in our area. The September conference was a small step towards recognising the importance of this area within the field of electroacoustic music studies. It is hoped that such events take place more often in the future demonstrating tangible results of today's and tomorrow's initiatives regarding widening interest and participation in our field.

Leigh Landy (editor - Organised Sound, conference and issue coordinator) (llandy@dmu.ac.uk) 